\title{
Aconiti Lateralis Radix Praeparata Lipid-Soluble Alkaloids Alleviates IL-1 $\beta$-Induced Inflammation of Human Fibroblast-Like Synoviocytes in Rheumatoid Arthritis by Inhibiting NF-KB and MAPKs Signaling Pathways and Inducing Apoptosis
}

\section{Chaocheng Guo}

Chengdu University of Traditional Chinese Medicine Wenjiang Campus: Chengdu University of

Traditional Chinese Medicine

\section{Linfeng He}

Chengdu University of Traditional Chinese Medicine Wenjiang Campus: Chengdu University of

Traditional Chinese Medicine

Naihua Hu

Chengdu University of Traditional Chinese Medicine Wenjiang Campus: Chengdu University of Traditional Chinese Medicine

\section{Xingtao Zhao}

Chengdu University of Traditional Chinese Medicine Wenjiang Campus: Chengdu University of Traditional Chinese Medicine

\section{Lihong Gong}

Chengdu University of Traditional Chinese Medicine Wenjiang Campus: Chengdu University of

Traditional Chinese Medicine

\section{Cheng Wang}

Chengdu University of Traditional Chinese Medicine Wenjiang Campus: Chengdu University of

Traditional Chinese Medicine

\section{Cheng Peng}

Chengdu University of Traditional Chinese Medicine Wenjiang Campus: Chengdu University of

Traditional Chinese Medicine

Yunxia Li (D lyxtgyxcdutcm@163.com)

Chengdu University of Traditional Chinese Medicine https://orcid.org/0000-0001-7211-1170

\section{Research}

Keywords: Aconiti Lateralis Radix Praeparata (Fuzi), Lipid-soluble alkaloids, Rheumatoid arthritis, Human fibroblast-like synoviocytes, Inflammation, Apoptosis 
Posted Date: September 2nd, 2021

DOI: https://doi.org/10.21203/rs.3.rs-855990/v1

License: (9) This work is licensed under a Creative Commons Attribution 4.0 International License. Read Full License

Version of Record: A version of this preprint was published at Cytokine on March 1st, 2022. See the published version at https://doi.org/10.1016/j.cyto.2022.155809. 


\section{Abstract}

Background: Fuzi lipid-soluble alkaloids (FLA) is the main bioactive components extracted from the traditional Chinese medicine Aconiti Lateralis Radix Praeparata ("Fuzi" in Chinese), which has promising analgesic and anti-inflammatory effects. However, the effects and the underlying mechanisms of FLA on rheumatoid arthritis (RA) have not been studied. The present study aimed to explore the anti-arthritic effects of FLA and its underlying mechanisms.

Methods: To standardize the FLA, UPLC-HR-MS was used for quantitative and qualitative analysis of the representative alkaloids. Cell viability was measured by MTT. The anti-inflammatory activity of FLA was examined by analyzing the expression levels of inflammatory mediators such as TNF-a, IL-6, MMP-1, MMP-3, PGE2, and COX-2 using ELISA and RT-PCR analysis. The Annexin V-FITC/PI double staining method was used to detect the apoptosis of HFLS-RA and analyzed by flow cytometry. Western blot analysis was used to analyze the expression of NF-KB, MAPKs and mitochondrial apoptosis pathway related proteins.

Results: FLA had a significant inhibitory effect on the proliferation of HFLS-RA induced by IL-1 $\beta$, which was accompanied by decreased expression levels of TNF-a, IL-6, MMP-1, MMP-3, COX-2 and PGE2. Remarkably, FLA inhibited the activation of NF-KB and MAPKs signaling pathways in IL-1 $\beta$-induced HFLSRA, as well as inducing HFLS-RA apoptosis through the mitochondrial apoptosis pathway.

Conclusions: FLA inhibited the expression and synthesis of inflammatory mediators by inhibiting the activation of NF-KB and MAPKs signaling pathways in HFLS-RA, and induced apoptosis of HFLS-RA via the mitochondrial apoptosis pathway.

\section{Introduction}

Rheumatoid arthritis (RA) is a chronic autoimmune inflammatory disease characterized by synovial inflammation and synovial tissue hyperplasia, which ultimately lead to the destruction of joint, cartilage and bone (Sparks, 2019). Approximately $0.5 \%-1 \%$ of the world's population is affected by RA, and its morbidity and mortality are rising with the increase in the population (Suzuki and Yamamoto, 2015).

Accumulating evidence indicate that fibroblast-like synoviocytes (FLS) plays a crucial role in RA progression. FLS can produce excessive inflammatory mediators, such as TNF- $a$ and IL-6 (Brzustewicz and Bryl, 2015), which trigger and perpetuate inflammation, and produce matrix metalloproteinases (MMPs) that contribute to cartilage and bone destruction, such as MMP-1 and MMP-3 (Murphy and Nagase, 2008). What's more, FLS also induces the production of the inflammatory mediator PGE2 by expressing COX-2, which is the major inflammatory mediator of rheumatoid arthritis and osteoarthritis (Choy and Panayi, 2001). Additionally, FLS is relatively resistant to apoptosis and forms hyperplastic synovial pannus tissue with macrophages, fibroblast-like mesenchymal cells and blood vessels (Bartok and Firestein, 2010). Therefore, inhibition of FLS-mediated synovial inflammation and synovial tissue hyperplasia may be a potential treatment for RA. 
It is widely reported that nuclear factor kappa B (NF-KB) and mitogen-activated protein kinases (MAPKs) signaling pathways are highly activated in the synovium of RA patients and mice with collagen-induced arthritis, playing a significant role in regulating synovial inflammation (Sun, 2017; Han et al., 2001). NF-kB is a transcription factor whose activity is mainly regulated by the interaction with inhibitory proteins, such as an inhibitor of kappa B (IKB). Inactivated NF-KB usually binds to IKB in the cytoplasm. When IKB degradation disrupts the interaction between IKB and NF-KB, NF-KB translocates to the nucleus and triggers the transcription and expression of pro-inflammatory cytokine mediators (Sun, 2017). MAPKs are serine-threonine kinases, which are mainly composed of c-Jun N-terminal kinase (JNK), p38 MAPK, and extracellular signal-regulated kinase 1/2 (ERK1/2). The MAPKs signaling pathway is primarily involved in direct cellular responses to various stressful stimuli, including inflammation (Ralph and Morand, 2008). Therefore, targeting synovial inflammation mediated by the NF-KB/MAPKs signaling pathway has become one of the important directions for the research of new anti-rheumatoid arthritis drugs.

Aconiti Lateralis Radix Praeparata ("Fuzi" in Chinese), the processed daughter or lateral root of Aconitum carmichaelii Debx (Fam. Ranunculaceae), is commonly used to treat collapse, diarrhea, syncope, rheumatic pain and paralysis joint pain, rheumatoid arthritis, and other inflammations (Nyirimigabo et al., 2015). Alkaloids are the main bioactive components in Fuzi, which can be divided into lipid-soluble alkaloids and water-soluble alkaloids according to their polarity. Fuzi lipid-soluble alkaloids (FLA) are mainly composed of monoester-diterpenoid aconitines (MDAs) and diester-diterpenoid aconitines (DDAs). The analgesic and anti-inflammatory effects of Fuzi are closely related to the existence of FLA (Bello-Ramírez et al., 2003). Previously, we found that the therapeutic dose of FLA $\left(12.5 \mathrm{mg} \cdot \mathrm{kg}^{-1} \cdot \mathrm{d}^{-1}\right)$ decreased the paws swelling and arthritis scores on adjuvant-induced arthritis (AIA) rats. In addition, plasma biochemical indexes also indicated that FLA could significantly inhibit the concentrations of IL$1 \beta$, TNF-a, and IFN- $\gamma$ on AIA rats (Xie et al., 2019). The above results illustrated that FLA possessed substantial therapeutic effects on AIA rats. However, the therapeutic mechanism of FLA on RA has not been studied, which seriously limits the clinical uses of FLA. Consequently, the purpose of this paper is to explore the anti-arthritic effects of FLA in IL-1 $\beta$-induced human fibroblast-like synoviocytes-rheumatoid arthritis (HFLS-RA) and its underlying pharmacological mechanisms.

\section{Materials And Methods 2.1 Preparation of FLA}

Fuzi was purchased from Sichuan Jiangyou Zhongba Fuzi Technology Development Co., Ltd. (Jiangyou, China), and was identified by professor Jin Pei from the department of pharmacognosy of Chengdu University of Traditional Chinese Medicine. In total, $5 \mathrm{~kg}$ of Fuzi was crushed into coarse powder and soaked in dichloromethane, and then transferred to $5 \% \mathrm{Na}_{2} \mathrm{CO}_{3}$ solution for $30 \mathrm{~min}$. Subsequently, the coarse powder of Fuzi was heating refluxed with dichloromethane $(1: 8, w / v)$ for $2 \mathrm{~h}$ at $65^{\circ} \mathrm{C}$. The filtrates were collected and the residues were then heating refluxed with dichloromethane $(1: 6, w / v)$ for $1 \mathrm{~h}$ at $65^{\circ} \mathrm{C}$. The two filtrates were mixed and concentrated to $2.5 \mathrm{~L}$. The concentrated solution was extracted 
twice with $0.05 \mathrm{~mol} / \mathrm{L} \mathrm{H}_{2} \mathrm{SO}_{4}(1: 1, \mathrm{v} / \mathrm{v})$ to obtain the acid solution, using ammonia to adjust $\mathrm{pH}$ to $9 \sim 10$, and then extracted with dichloromethane $(1: 1, v / v)$ for 3 times. Finally, the extract was combined and dichloromethane was retrieved to obtain about $12.5 \mathrm{~g}$ of FLA.

\subsection{UPLC-HR-MS analysis of FLA}

The ultra-high performance liquid chromatography-high resolution-mass spectrometry was used for qualitative and quantitative analysis of FLA. A BEH C18 column $(1.7 \mu \mathrm{m}, 2.1 \mathrm{~mm}$ ID×50 mm, Waters, Milford, MA, USA) was used for the separation of FLA. The column temperature was maintained at $35^{\circ} \mathrm{C}$. The mobile phase was acetonitrile $(A)$ mixed with water $(0.1 \%$ formic acid, $B)$ in gradient mode at a flow rate of $300 \mu \mathrm{L} / \mathrm{min}$. The elution gradient was optimized as follows: $0-5 \mathrm{~min}, 80-0 \% \mathrm{~B} ; 5-7 \mathrm{~min}, 100 \% \mathrm{~A}$; 7-8 min, $0-80 \% \mathrm{~B} ; 8-10 \mathrm{~min}, 80 \% \mathrm{~B}$. The injection volume was $0.2 \mu \mathrm{L}$ and the sample was set at $4{ }^{\circ} \mathrm{C}$. MassLynx software was used to manage UPLC-HR-MS and collect and analyze data.

Aconitine (AC), mesaconitine (MA) and hypoaconitine (HA) were used as reference compounds for FLA analysis, and they were supplied from Chengdu Chroma Biotechnology Co., Ltd. (Chengdu, China). The UPLC-HR-MS chromatogram of the FLA is shown in Fig. 1. The contents of AC, MA and HA in FLA were $0.264 \%(2.64 \mathrm{mg} / \mathrm{g}), 8.24 \%(82.4 \mathrm{mg} / \mathrm{g})$, and $1.08 \%(10.8 \mathrm{mg} / \mathrm{g})$, respectively.

\subsection{Cell culture}

The Primary HFLS-RA were purchased from Guangzhou Otwobiotech Co., Ltd. (Guangzhou, China), and cultured in DMEM supplemented with $10 \%$ fetal bovine serum, $1 \%$ penicillin/streptomycin under a humidified atmosphere $5 \% \mathrm{CO}_{2}$ at $37^{\circ} \mathrm{C}$. The HFLS-RA at passages 3-9 were used for all experiments. FLA was dissolved in dimethyl sulfoxide (DMSO) and diluted to the required concentration with a serumfree medium. The final concentration of DMSO was less than $0.1 \%$, which did not effect on cell viability.

\subsection{Cell viability assay}

HFLS-RA cells were seeded into $96-$ well plates at the density of $5 \times 10^{3} /$ well and incubated for $12 \mathrm{~h}$. The medium was removed and fresh serum-free medium with or without various kinds of reagents was added. At the end of incubation, $20 \mu \mathrm{L}$ MTT (Solarbio, Beijing, China) solution was added to each well and further incubated $4 \mathrm{~h}$ at $37^{\circ} \mathrm{C}$. Then incubation was stopped and $150 \mu \mathrm{L}$ DMSO was added to each well to dissolve MTT formazan crystals. The optical density was detected with microplate reader at $490 \mathrm{~nm}$. The cell viability was calculated using $\left(\mathrm{OD}_{\text {experiment }}-\mathrm{OD}_{\text {blank }}\right) /\left(\mathrm{OD}_{\text {control }}-\mathrm{OD}_{\text {blank }}\right) \times 100 \%$.

\subsection{Enzyme-linked immunosorbent assay (ELISA)}

HFLS-RA cells were seeded into 6-well plates at the density of $1 \times 10^{5} /$ well and incubated for $12 \mathrm{~h}$. The cells were exposed to different concentrations of FLA for 1 hour and then treated with IL-1 $\beta$ for another 24 hours. At the end of incubation, the cell supernatant was collected. Subsequently, the IL-6, TNF-a, MMP-1, MMP-3 and PGE2 were analyzed by ELISA kits (Multi Sciences, Hangzhou, China).

\subsection{RT-qPCR}


Total RNA from HFLS-RA was extracted using the Trizol Reagent following the manufacturer's protocols. Total RNA was reverse transcribed into cDNA using the 5X All-In-One MasterMix (abm, Canada).

Quantitative PCR was performed on the Applied Biosystems $7900 \mathrm{HT}$ fast system using SYBR Green PCR Master Mix (Thermo, Hudson, NH, USA). Expression data were calculated according to the amplification curve by using the $2^{-\Delta \Delta C t}$ method. Primers sequences used were provided in Table 1.

Table 1

Primers used for RT-PCR in this study.

\begin{tabular}{|l|lll|}
\hline Gene & Forward (5'-3') & Reverse (5'-3') \\
\hline TNF-a & CACAGTGAAGTGCTGGCAAC & GATCAAAGCTGTAGGCCCCA \\
IL-6 & ACAGGGAGAGGGAGCGATA & CCAGTCCTCTTTGTTGGGGAT \\
MMP-1 & CAGAGATGAAGTCCGGTTTTTC & GGGGTATCCGTGTAGCACAT \\
MMP-3 & TCAAGGGAACTTGAGCGTGA & AAGCTAAGCAGCAGCCCATT \\
COX-2 & AGGGTTGCTGGTGGTAGGAA & CATCTGCCTGCTCTGGTCAA \\
GAPDH & TGGAAGGACTCATGACCACA & RAGGGGTCTACATGGCAACTG \\
\hline Aptosis assay &
\end{tabular}

HFLS-RA cells were seeded into 6 -well plates at the density of $1 \times 10^{5} /$ well and incubated for $12 \mathrm{~h}$. The cells were exposed to different concentrations of FLA for 1 hour and then treated with IL-1 $\beta$ for another 24 hours. By then, the cells were collected by centrifugation at $800 \times \mathrm{g}$ for 10 minutes, and washed three times with precooled phosphate buffer saline (PBS). According to the introduction of the Annexin VFITC/PI Apoptosis Detection Kit (Multi Sciences, Hangzhou, China), the cells were resuspended with 500 $\mu \mathrm{L}$ of binding buffer and incubated with $5 \mu \mathrm{L}$ of Annexin V-FITC and $10 \mu \mathrm{L}$ of PI for 5 minutes at room temperature in the dark. Apoptotic cells were detected by utilizing a FACSCalibur flow cytometer (BD Biosciences, San Jose, CA).

\subsection{Western blot assay}

HFLS-RA cells were cultivated in 60-mm petri dishes and washed twice with precooled PBS after stimulation as described above. The cell lysate was prepared by adding protease and phosphatase inhibitors to the RIPA buffer. After cell lysis, the supernatant was collected by centrifugation at $12 \times 10^{3}$ rpm for 15 minutes, and then protein concentration was quantified by using BCA Kit (Multi Sciences, Hangzhou, China). Equal amounts of samples were separated by $10 \%$ SDS-PAGE and then transferred onto PVDF membranes. After that, the PVDF membranes were blocked with TBST containing $5 \%$ non-fat milk for $2 \mathrm{~h}$. Subsequently, the membranes were incubated with primary antibodies (rabbit polyclonal anti Bax, Bcl-2, Cytochrome C, Cleaved caspase3, cleaved caspase 9, Cleaved PARP, ERK1/2, p-ERK1/2, p38, pp38, JNK, p-JNK, p65, IKBa, Histone H3 and $\beta$-actin; 1:500-1:1000) at $4^{\circ} \mathrm{C}$ overnight and with secondary antibodies (Goat anti-Rabbit IgG-HRP; $1: 5000$ ) at $37^{\circ} \mathrm{C}$ for a further $2 \mathrm{~h}$. Finally, the ECL Kit (Amersham, 
Arlington Heights, IL, USA) was used to visualize protein bands, and the immunoblot signal was quantificationally analyzed with Image $\mathrm{J}$ software.

\subsection{Statistical analysis}

Statistical analysis was performed using SPSS 25.0 software and data were expressed as Mean \pm SD. One-Way ANOVA was used to evaluate the statistical differences among the multiple groups. P-value< 0.05 was considered as statistically significant.

\section{Results}

\subsection{IL-1 $\beta$ stimulated the proliferation of HFLS-RA and promoted the expression of Inflammatory mediators in HFLS-RA}

Previous studies have reported that IL-1 $\beta$-induced HFLS-RA model was used to study the drugs for treatment of RA in vitro. To explore the modeling concentration of IL-1 $\beta$, the effects of different concentrations of IL-1 $\beta$ on the proliferation of HFLS-RA, the expression of inflammatory mediators were investigated. As shown in Fig. $2 A$, IL-1 $\beta$ significantly increased the cell viability of HFLS-RA with the increase of dose, suggesting that IL-1 $\beta$ could induce the proliferation of HFLS-RA. Consistently, IL-1 $\beta$ significantly induced the expression of IL-6, TNF-a, MMP-1, MMP-3 and PGE2 (Fig. 2B-F), indicating that IL-1 $\beta$ could also induce the inflammatory response of HFLS-RA. However, $5 \mathrm{ng} / \mathrm{mL}$ of IL-1 $\beta$ could not significantly induce the expression of MMP-1 in HFLS-RA. Based on the above results, $10 \mathrm{ng} / \mathrm{mL}$ of IL-1 was selected to establish the HFLA-RA model.

\subsection{FLA inhibited IL-1 $\beta$-induced proliferation of HFLS-RA}

Primarily, to ensure medication safety, we tested the potential cytotoxic effects of FLA on HFLS-RA. As shown in Fig. 3A, FLA $(0.1 \mathrm{mg} / \mathrm{mL})$ had no obvious effect on the cell viability of HFLS-RA at $12 \mathrm{~h}$ and 24 $\mathrm{h}$ but not $48 \mathrm{~h}$. To investigate the possible inhibitory effects of FLA on IL-1 $\beta$-induced HFLS-RA proliferation, HFLS-RA was incubated with IL-1 $\beta$ in the presence or absence of FLA for $24 \mathrm{~h}$. As shown in Fig. 3B, we observed that IL-1 $\beta$ significantly improved the cell viability of HFLS-RA compared with the untreated control group. FLA $(0.025,0.05$ and $0.1 \mathrm{mg} / \mathrm{mL})$ treatment significantly reversed the observed viability increase induced by $\mathrm{IL}-1 \beta$. Therefore, $0.025 \mathrm{mg} / \mathrm{mL}, 0.05 \mathrm{mg} / \mathrm{mL}, 0.1 \mathrm{mg} / \mathrm{mL}$ of FLA were selected as the final dose concentration for further investigation of the mechanisms of the FLA in IL-1 $\beta$ induced HFLS-RA.

\subsection{FLA inhibited IL-1 $\beta$-induced expression of inflammatory mediators in HFLS-RA}


The effect of FLA on IL-1 $\beta$-induced inflammation in HFLS-RA was investigated by ELISA and RT-PCR experiment. As shown in Fig. 4, IL-1 $\beta$ stimulation significantly enhanced the protein levels of IL-6, TNF-a and PGE2 and the mRNA levels of IL- 6 , TNF- $a$ and COX-2 compared with untreated HFLS-RA. FLA obviously downregulated IL-1 $\beta$-induced increases of IL-6, TNF-a, PGE2 and COX-2 expression in HFLS-RA. However, at $25 \mu \mathrm{g} / \mathrm{mL}$, FLA had no significant effect on the expression of FGE2 and MMP-1 compared with IL-1 $\beta$-induced group. Moreover, as shown in Fig. 5, IL-1 $\beta$ stimulation significantly enhanced the protein and mRNA levels of MMP-1 and MMP-3, which was reversed by FLA treatment.

\subsection{FLA inhibited IL-1 $\beta$-induced activation of NF-KB and MAPKs signaling pathways in HFLS-RA}

Previous studies have shown that NF-KB and MAPKs signaling pathways are significantly activated in the pathogenesis of RA. Thus, we wondered whether FLA inhibits the expression of inflammatory mediators in IL-1 $\beta$-induced HFLS-RA by regulating these transduction pathways. As expected, IL-1 $\beta$ stimulation obviously induced the phosphorylation of ERK1/2, JNK and p38 compared with control group. FLA significantly inhibited the phosphorylation of ERK1/2, JNK and p38 induced by IL-1 $\beta$, but FLA $(25 \mu \mathrm{g} / \mathrm{ml})$ had no significant effect on the phosphorylation of p38 (Fig. 6A). As shown in Fig. 6B, IL-1 $\beta$ stimulation significantly induced the nuclear translocation of NF-KB/p65 and the degradation of cytoplasmic $1 \mathrm{KBa}$, which was reversed by FLA treatment. These results suggested that FLA might inhibit IL-1 $\beta$-induced the expression of inflammatory mediators via intracellular NF-KB and MAPKs pathways.

\subsection{FLA induced apoptosis in HFLS-RA}

Inducing apoptosis of hyperproliferative HFLS-RA is an effective method to treat RA. Thus, Annexin VFITC/PI double staining was used to assess the apoptosis of HFLS-RA. As shown in Fig. 7, IL-1 $\beta$ stimulation obviously inhibited the apoptosis of HFLS-RA compared with control group. FLA significantly induced the apoptosis of HFLS-RA, including early apoptotic and late apoptotic cells.

\subsection{FLA regulated the expression levels of mitochondrial apoptosis pathway-related proteins in HFLS-RA}

Mitochondria plays a key role in cellular apoptosis. Hence, to further clarify the potential molecular mechanisms of FLA-induced apoptosis of HFLS-RA, western blotting was used to detect the changes of relative apoptotic proteins in HFLS-RA mitochondrial apoptosis pathway after $12 \mathrm{~h}$ of FLA stimulation. As shown in Fig. 8, FLA significantly promoted the expression of pro-apoptotic protein Bax, but significantly inhibited the expression of anti-apoptotic protein Bcl-2 in HFLS-RA. Bax can form mitochondrial permeablity transition pore (MPTP), leading to the release of cytochrome $C$ into the cytoplasm, thereby activating the caspase cascade. Finally, the caspase cascade leads to apoptosis. Consistently, FLA also significantly promoted the expression of Cytochrome C, cleaved caspase-3, cleaved caspase-9 and cleaved PARP (Fig. 8). Combining with the results of Annexin V-FITC/PI double staining and MTT assay, 
we speculated that FLA might induce HFLS-RA apoptosis via the mitochondrial apoptotic pathway, thereby inhibiting hyperproliferation of HFLS-RA.

\section{Discussion}

The clinical symptoms of RA are bilateral symmetrical polyarthritis, which is an important cause of musculoskeletal disability. Currently, the available drugs for the treatment of RA include non-steroidal anti-inflammatory drugs (NSAIDs) targeting COX-2, immunosuppressive glucocorticoids and diseasemodifying anti-rheumatic drugs (DMARDs), inhibiting the proliferation of pannus tissues (Lin et al., 2020). With the development of anti-rheumatic drugs, rheumatoid arthritis has become a manageable disease. However, the development of effective, safe and low-cost medicines for RA is still the top priority of RA research at the present stage.

FLS, as a significant component of invasive rheumatoid synovium, is the key pro-inflammatory cells that promote the progression of RA. This suggests that targeting FLS may provide a new class of therapeutic agents for the treatment of RA. As the predominant inflammatory cytokine associated with RA, IL-1 $\beta$ has been widely used to establish RA model in vitro studies. However, the modeling concentration of IL-1 $\beta$ has not been reported yet. In the current study, we found that $10 \mathrm{ng} / \mathrm{ml} \mathrm{IL-1 \beta}$ could successfully establish RA model in vitro.

The activated FLS can synthesize pro-inflammatory mediators with a variety of diverse functions by activating multiple signaling pathways. These pro-inflammatory mediators further stimulate FLS and other cells in the joint environment in autocrine or paracrine fashion, leading to increased inflammation and joint destruction. Especially, TNF- $a$ and IL- 6 produced by FLS are important pro-inflammatory cytokines of synovial inflammation in RA. Moreover, TNF- $\alpha$ and IL- 6 also both initiate and perpetuate the production of MMPs (Reboul et al., 1996) and prostaglandins (Dayer et al., 1996). Among of them, MMP1 and MMP-3 cause irreversible destruction of cartilage, bone and tendons in the joints, which play a leading role in the degradation of the articular matrix. Meanwhile, FLS is considered to be the main cellular source of COX-2 in synovial tissue and produce PGE2, which mediates the pain and inflammation in RA (Amin et al., 1999). Notably, in previous research, we have demonstrated that FLA significantly alleviated the symptoms of arthritis in AIA mice (Xie et al., 2019). This study found that FLA effectively inhibited the expression and synthesis of pro-inflammatory mediators in IL-1 $\beta$-induced HFLS-RA.

The NF-KB signaling pathway not only regulates numerous inflammatory genes, but also controls the expression of several MMPs (Mengshol et al., 2000). The promoters of MMP-1, MMP-3 and MMP-6 contain typical binding sites of NF-KB, and the activation of NF-KB can induce the expression of MMPmRNA by targeting the promoter region of related MMPs. In addition, the MAPKs signaling pathway targets AP-1 and Ets sites in the MMP promoter to regulate the expression of MMPs genes (Mengshol et al., 2002). Numerous studies have demonstrated that inhibition of NF-KB or MAPKs signaling pathways can reduce the expression and synthesis of pro-inflammatory mediators in models of arthritis in vivo and in vitro (Mclntyre et al., 2003; Lee et al., 2014). To further explore the possible pharmacological 
mechanisms of FLA on the expression and synthesis of pro-inflammatory mediators in IL-1 $\beta$-induced HFLS-RA, the activation of NF-KB and MAPKs was detected. This study showed that FLA significantly inhibited the IL-1 $\beta$-induced activation of NF-KB and phosphorylation of MAPKs. The results proved that FLA exerted anti-inflammatory effects by inhibiting the activation of NF-KB and MAPKs in HFLS-RA.

FLS activation leads to the hyperplastic synovial lining layer, which may be caused by a variety of reasons, including FLS proliferation, reduced apoptosis, and recruitment from other fibroblast pools, etc. The hyperplastic synovial lining layer invades the periarticular bone at the cartilage-bone junction by transforming into pannus, leading to bone erosion and cartilage degeneration (Aletaha and Smolen, 2018). Therefore, therapies aimed at inhibiting the excessive proliferation of HFLS-RA and inducing HFLS-RA apoptosis are meaningful for the treatment of RA. A previous study had reported that FLA can significantly induce apoptosis of nerve cells in juvenile zebrafish (Li et al., 2019). In the present study, MTT assay showed that FLA had significant inhibition effect on IL-1 $\beta$-induced HFLS-RA proliferation. Meanwhile, the effect of FLA on the apoptosis of HFLS-RA was further examined, and Annexin V-FITC/PI double staining showed that FLA induced apoptosis of HFLS-RA. Moreover, to further explore the possible pharmacological mechanisms of FLA-induced apoptosis of HFLS-RA, the expression levels of mitochondrial apoptosis pathway-related proteins were measured. In this study, we found that FLAinduced apoptosis of HFLS-RA may be achieved through the endogenous mitochondrial-dependent pathway.

This study had several limitations. Firstly, it is generally believed that FLA have high toxicity, especially the DDAs represented by AC, MA and HA, which can cause acute cardiac toxicity, neurotoxicity and reproductive toxicity, etc. However, modern studies have shown that FLA can exert pharmacological efficacy at low concentrations. How to reduce the toxicity of FLA as much as possible on the basis of ensuring its efficacy still needs further exploration. Secondly, although the mechanisms of FLA induced apoptosis of HFLS-RA have been proved to be related to mitochondrial apoptosis pathway, further studies are still needed to determine whether other signaling pathways are involved in this process. For example, both TNF-a stimulation and NF-KB activation decrease FLS apoptosis through mechanisms, including resistance to Fas ligand-induced signals and up-regulation of anti-apoptotic molecules such as Bcl-2 (Baier et al., 2003). In this study, we found that FLA can inhibit the synthesis of TNF- $a$ and the activation of NF-KB, which indicates that NF-KB pathway may also be involved in FLA-induced apoptosis of HFLSRA. However, the correlation between the NF-KB signaling pathway and the mitochondrial apoptosis pathway remains to be elucidated.

\section{Conclusions}

The research results provided the first evidence that FLA can inhibit IL-1 $\beta$-induced the expression of inflammatory mediators in HFLS-RA and induce HFLS-RA apoptosis. The possible molecular mechanisms of FLA on HFLS-RA may be closely related with inhibition of NF-KB, MAPKs and activation of mitochondrial apoptosis pathways. 


\section{Abbreviations}

DMARD, disease-modifying antirheumatic drug; ERK, extracellular signal-regulated kinase; Fuzi, Aconiti Lateralis Radix Praeparata; FLA, Aconiti Lateralis Radix Praeparata lipid-soluble alkaloids; GAPDH, glyceraldehyde 3-phosphate dehydrogenase; HFLS-RA, human fibroblast-like synoviocytes-rheumatoid arthritis; JNK, c-Jun N-terminal kinase; MAPKs, mitogen-activated protein kinases; MMP, matrix metalloproteinase; NSAID, non-steroidal anti-inflammatory drug; RA, rheumatoid arthritis.

\section{Declarations}

\section{Ethics approval and consent to participate}

All the procedures were approved by the Animal Ethics Committee of Chengdu University of Traditional Chinese Medicine before the experiment.

\section{Consent for publication}

Not applicable.

\section{Availability of data and materials}

The datasets used and/or analysed during the current study are available from the corresponding author on reasonable request.

\section{Competing interests}

The authors declare that they have no competing interests.

\section{Author contributions}

Chaocheng Guo, Yunxia Li and Cheng Peng designed the study and directed the study's optimization strategy. Chaocheng Guo summarized the Results and Discussion sections of the text. WB and RT-PCR were performed by Linfeng He, Naihua Hu, Xingtao Zhao, Lihong Gong and Cheng Wang conducted the biological experiments and analyzed the data. All the authors read and approved the final manuscript.

\section{Acknowledgements}

We really appreciate the technological support from team of Cheng Peng (Chengdu University of Traditional Chinese Medicine).

\section{Funding}

The study was supported by the National Science Foundation of China (Grant No. 81891012, 8163010, U19A2010), Sichuan Science and Technology Program (Grant No. 2021JDRC0041), Xinglin Scholar Research Premotion Project of Chengdu University of TCM (Grant No. CXTD2018019). 


\section{References}

Amin, A.R., Attur, M., Abramson, S.B., 1999. Nitric oxide synthase and cyclooxygenases: distribution, regulation, and intervention in arthritis. Curr Opin Rheumatol. 11 (3), 202-209. doi: 10.1097/00002281199905000-00009.

Aletaha, D., Smolen, J.S., 2018. Diagnosis and Management of Rheumatoid Arthritis: A Review. JAMA. 320 (13), 1360-1372. doi: 10.1001/jama.2018.13103.

Baier, A., Meineckel, I., Gay, S., Pap, T., 2003. Apoptosis in rheumatoid arthritis. Curr Opin Rheumatol. 15 (3), 274-279. doi: 10.1097/00002281-200305000-00015.

Bello-Ramírez, A.M., Buendía-Orozco, J., Nava-Ocampo, A.A., 2003. A QSAR analysis to explain the analgesic properties of Aconitum alkaloids. Fundam Clin Pharmacol. 17 (5), 575-580. doi: 10.1046/j.1472-8206.2003.00189.x.

Bartok, B., Firestein, G.S., 2010. Fibroblast-like synoviocytes: key effector cells in rheumatoid arthritis. Immunol Rev. 233 (1), 233-255. doi: 10.1111/j.0105-2896.2009.00859.x.

Brzustewicz, E., Bryl, E., 2015. The role of cytokines in the pathogenesis of rheumatoid arthritis-Practical and potential application of cytokines as biomarkers and targets of personalized therapy. Cytokine. 76 (2), 527-536. doi: 10.1016/j.cyto.2015.08.260. Epub 2015 Aug 29.

Choy, E.H., Panayi, G.S., 2001. Cytokine pathways and joint inflammation in rheumatoid arthritis. N Engl J Med. 344 (12), 907-916. doi: 10.1056/NEJM200103223441207.

Dayer, J.M., Beutler, B., Cerami, A., 1985. Cachectin/tumor necrosis factor stimulates collagenase and prostaglandin E2 production by human synovial cells and dermal fibroblasts. J Exp Med. 162 (6), 21632168. doi: 10.1084/jem.162.6.2163.

Han, Z., Boyle, D.L., Chang, L., Bennett, B., Karin, M., Yang, L., Manning, A.M., Firestein, G.S., 2001. c-Jun Nterminal kinase is required for metalloproteinase expression and joint destruction in inflammatory arthritis. J Clin Invest. 108 (1), 73-81. doi: 10.1172/JCI12466.

Lee, W.S., Lim, J.H., Sung, M.S., Lee, E.G., Oh, Y.J., Yoo, W.H., 2014. Ethyl acetate fraction from Angelica sinensis inhibits IL-1 $\beta$-induced rheumatoid synovial fibroblast proliferation and COX-2, PGE2, and MMPs production. Biol Res. 47 (1), 41. doi: 10.1186/0717-6287-47-41.

Li, M.T., Zhang, H.Q., Wen, R.Q., Xiang, Y.S., Peng, C., Xie, X.F., 2019. Study on the neurotoxicity of lipidsoluble alkaloids from Aconiti Lateralis Radix Praeparata based on zebrafish. Pharmacology and Clinics of Chinese Materia Medica. 35 (06), 63-66.

Lin, Y.J., Anzaghe, M., Schülke, S., 2020. Update on the Pathomechanism, Diagnosis, and Treatment Options for Rheumatoid Arthritis. Cells. 9(4), 880. doi: 10.3390/cells9040880. 
Mengshol, J.A., Vincenti, M.P., Coon, C.I., Barchowsky, A., Brinckerhoff, C.E., 2000. Interleukin-1 induction of collagenase 3 (matrix metalloproteinase 13) gene expression in chondrocytes requires p38, c-Jun $\mathrm{N}$ terminal kinase, and nuclear factor kappaB: differential regulation of collagenase 1 and collagenase 3 . Arthritis Rheum. 43 (4), 801-811. doi: 10.1002/1529-0131(200004)43:4<801::AID-ANR10>3.0.C0;2-4.

Mengshol, J.A., Mix, K.S., Brinckerhoff, C.E., 2002. Matrix metalloproteinases as therapeutic targets in arthritic diseases: bull's-eye or missing the mark? Arthritis Rheum. 46 (1), 13-20. doi: 10.1002/15290131(200201)46:1<13::aid-art497>3.0.co;2-s.

McIntyre, K.W., Shuster, D.J., Gillooly, K.M., Dambach, D.M., Pattoli, M.A., Lu, P., Zhou, X.D., Qiu,Y., Zusi, F.C., Burke, J.R., 2003. A highly selective inhibitor of I kappa B kinase, BMS-345541, blocks both joint inflammation and destruction in collagen-induced arthritis in mice. Arthritis Rheum. 48 (9), 2652-2659. doi: 10.1002/art.11131.

Murphy, G., Nagase, H., 2008. Progress in matrix metalloproteinase research. Mol Aspects Med. 29 (5), 290-308. doi: 10.1016/j.mam.2008.05.002.

Nyirimigabo, E., Xu, Y., Li, Y., Wang, Y., Agyemang, K., Zhang, Y., 2015. A review on phytochemistry, pharmacology and toxicology studies of Aconitum. J Pharm Pharmacol. 67 (1), 1-19. doi: 10.1111/jphp.12310.

Reboul, P., Pelletier, J.P., Tardif, G., Cloutier, J.M., Martel-Pelletier, J., 1996. The new collagenase, collagenase-3, is expressed and synthesized by human chondrocytes but not by synoviocytes. A role in osteoarthritis. J Clin Invest. 97 (9), 2011-2019. doi: 10.1172/JCI118636.

Ralph, J.A., Morand, E.F., 2008. MAPK phosphatases as novel targets for rheumatoid arthritis. Expert Opin Ther Targets. 12 (7), 795-808. doi: 10.1517/14728222.12.7.795.

Suzuki, A., Yamamoto, K., 2015. From genetics to functional insights into rheumatoid arthritis. Clin Exp Rheumatol. 33 (4 Suppl 92), S40-S43.

Sun, S.C., 2017. The non-canonical NF-kB pathway in immunity and inflammation. Nat Rev Immunol. 17 (9), 545-558. doi: 10.1038/nri.2017.52.

Sparks, J.A., 2019. Rheumatoid Arthritis. Ann Intern Med. 170 (1), ITC1-ITC16. doi: 10.7326/AITC201901010.

Xie, Y.F., Feng, W.W., Liu, M.C., Xie, J., Yu, L., Gong, X.H., Li, Y.X., Peng, C., 2019. Investigation of Efficacy Enhancing and Toxicity Reducing Mechanism of Combination of Aconiti Lateralis Radix Praeparata and Paeoniae Radix Alba in Adjuvant-Induced Arthritis Rats by Metabolomics. Evid Based Complement Alternat Med. 9864841. doi: 10.1155/2019/9864841.

\section{Figures}



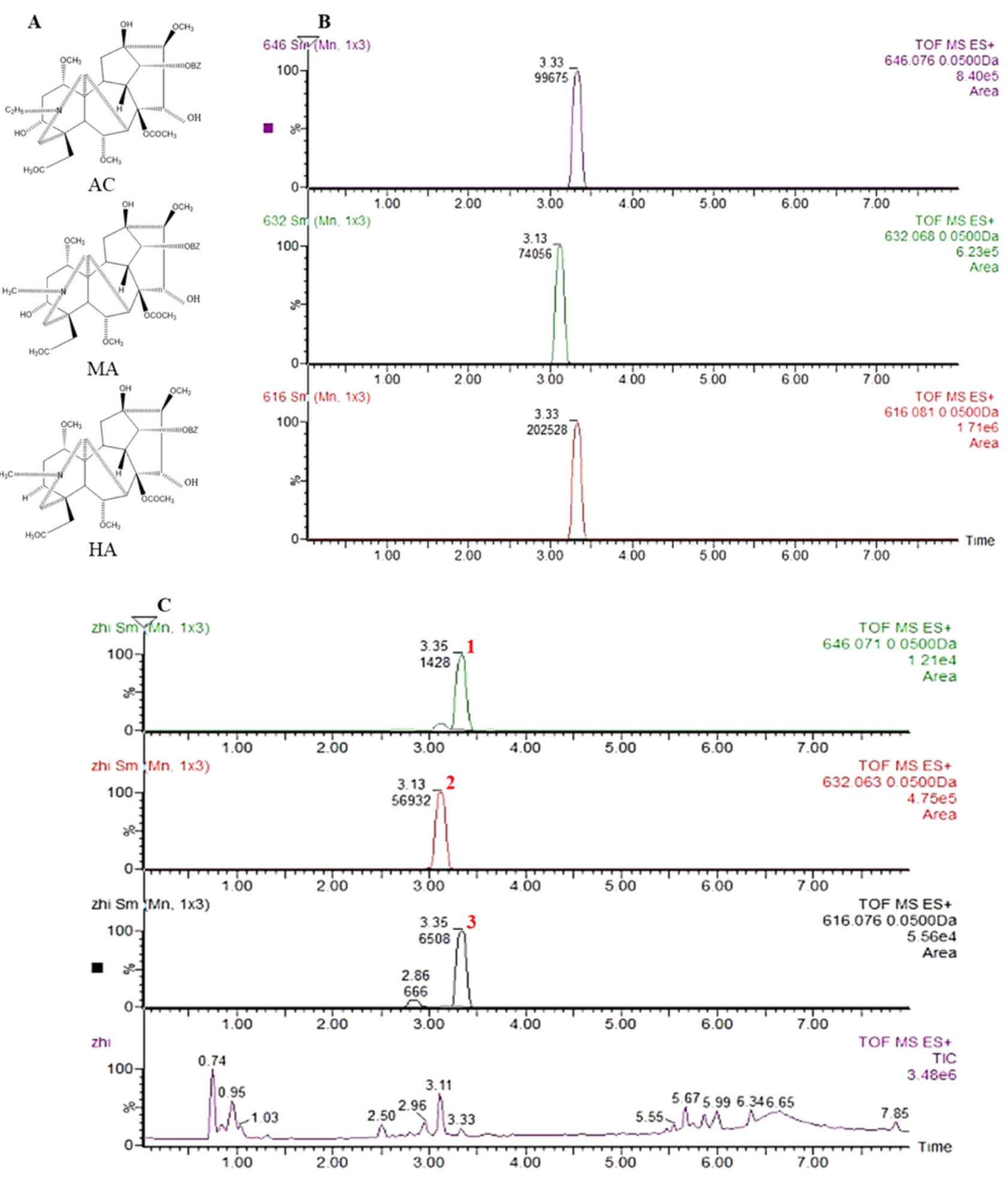

\section{Figure 1}

The analysis results of FLA by UPLC-HR-MS. (A) The chemical structures of AC, MA and HA. (B) The reference substance of AC, MA and HA in UPLC-HR-MS. (C) The UPLC-HR-MS analysis results of FLA. 1. AC. 2. MA. 3. HA. 
Figure 2

(A) Effects of different IL-1 $\beta$ concentrations on cell viability of HFLS-RA. (B-F) Effects of different IL-1 $\beta$ concentrations on the expression of IL-6, TNF- $a$, MMP-1, MMP-3 and PGE2 in HFLS-RA. HFLS-RA were incubated with various concentrations of IL-1 $\beta$ for $24 \mathrm{~h}$. Values are expressed as means \pm SD of three independent experiments. ${ }^{* \star} p<0.001 \mathrm{IL}-1 \beta$ vs control, ${ }^{* *} p<0.01 \mathrm{IL}-1 \beta$ vs control and ${ }^{*} p<0.05 \mathrm{IL}-1 \beta$ vs control.
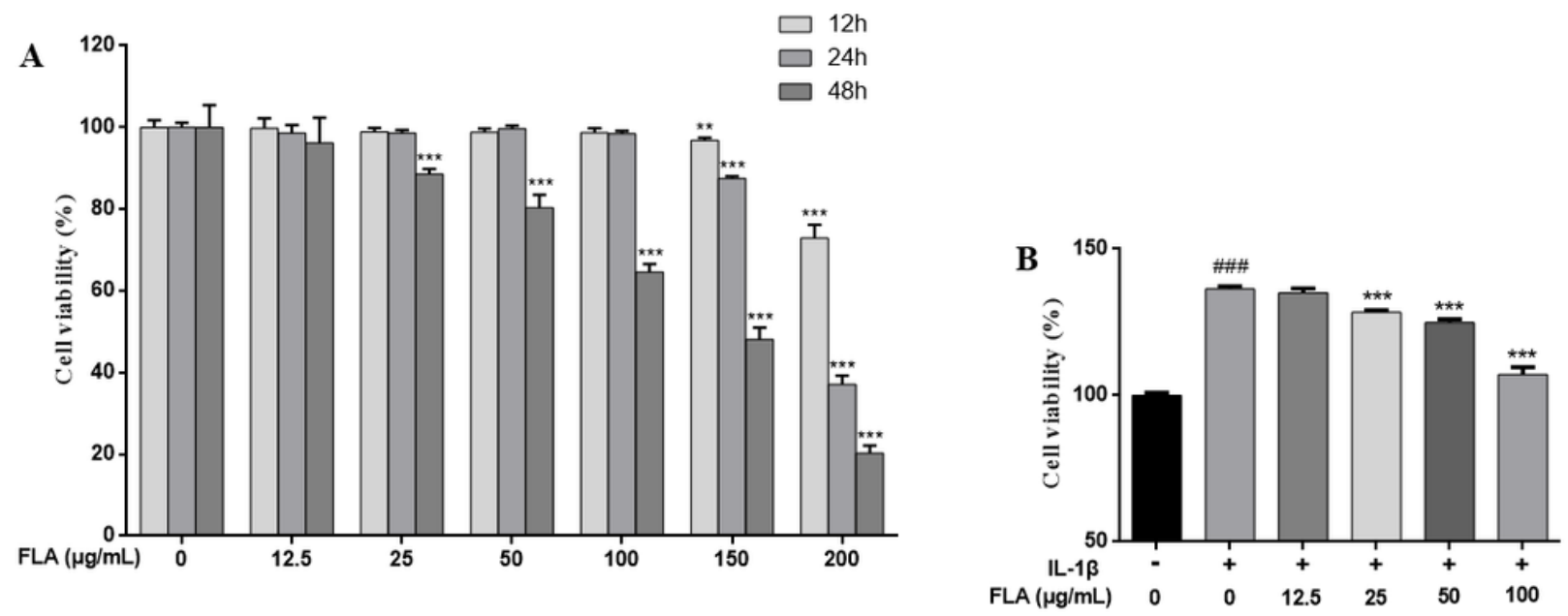

Figure 3

Effects of FLA on cell viability of HFLS-RA with or without IL-1 $\beta$ stimulation. (A) HFLS-RA were seeded in a 96-well plate and incubated with various concentrations of FLA for $12 \mathrm{~h}, 24 \mathrm{~h}$ and $48 \mathrm{~h}$. (B) HFLS-RA were pretreated with various concentrations of FLA for $1 \mathrm{~h}$ and then exposed to IL-1 $\beta$ for another $24 \mathrm{~h}$. Values are expressed as means \pm SD of three independent experiments. \#\#\# $p<0.001 \mathrm{IL}-1 \beta$ vs control, $\star * \star p<0.001 \mathrm{IL}-1 \beta+$ FLA vs IL-1 $\beta$ and $* * p<0.01 \mathrm{IL}-1 \beta+$ FLA vs IL-1 $\beta$. 

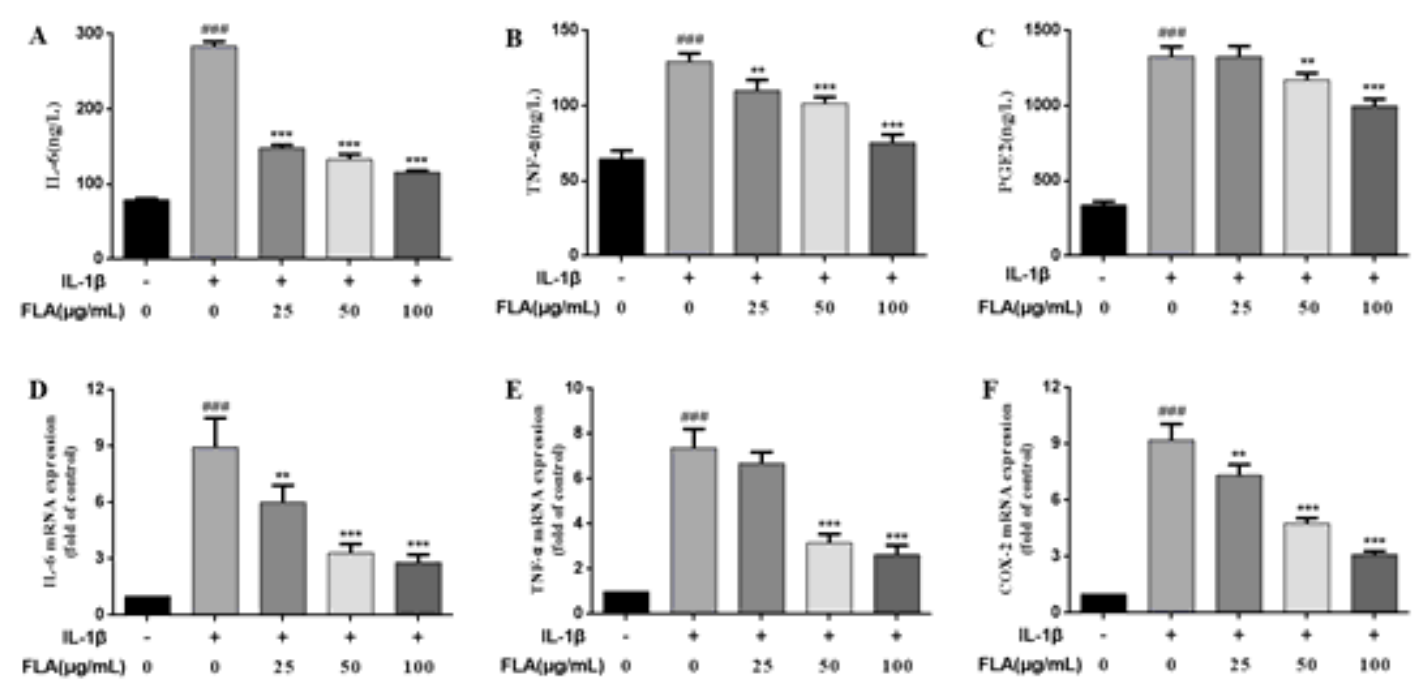

\section{Figure 4}

Effects of FLA on IL-6, TNF-a, PGE2 and COX-2 protein and mRNA levels in HFLS-RA. (A, B, and C) The protein levels of IL-6, TNF- $a$ and PGE2 in the culture supernatants were detected by ELISA. (D, E and F) The mRNA levels of IL-6, TNF- $a$ and COX-2 were analyzed using RT-PCR assay. Values are expressed as means \pm SD of three independent experiments. \#\#\# $p<0.001 \mathrm{IL}-1 \beta$ vs control, $* \star \star ~ p<0.001 \mathrm{IL}-1 \beta+F L A$ vs IL-1 $\beta$ and ** $p<0.01 \mathrm{IL}-1 \beta+$ FLA vs IL-1 $\beta$.
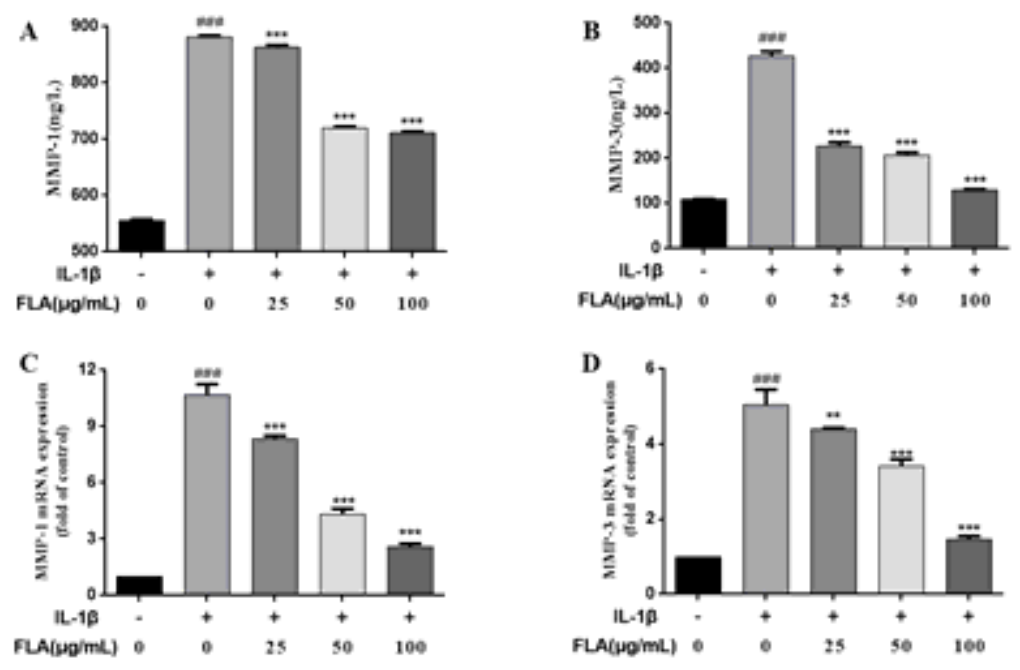

Figure 5

Effects of FLA on MMP-1 and MMP-3 protein and mRNA levels in HFLS-RA. (A and B) The protein levels of MMP-1 and MMP-2 in the culture supernatants were detected by ELISA. ( $C$ and $D$ ) The mRNA level of MMP-1 and MMP-2 was analyzed using RT-PCR assay. Values are expressed as means \pm SD of three 
independent experiments. \#\#\# $p<0.001 \mathrm{IL}-1 \beta$ vs control, $* \star \star p<0.001 \mathrm{IL}-1 \beta+$ FLA vs IL-1 $\beta$ and $* \star p<$ $0.01 \mathrm{IL}-1 \beta+\mathrm{FLA}$ vs IL-1 $\beta$.

A
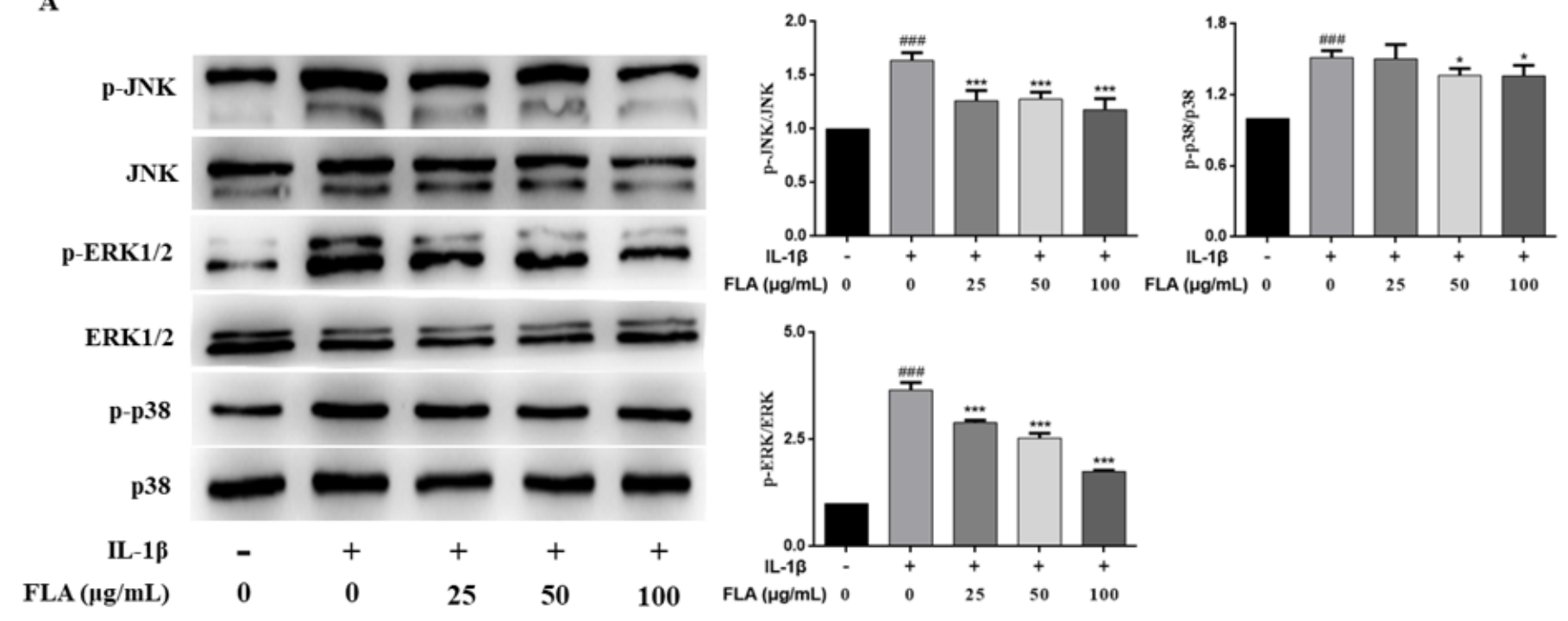

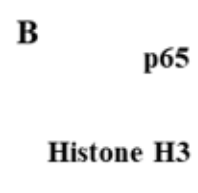

IL-1及 FLA $(\mu \mathrm{g} / \mathrm{mL})$
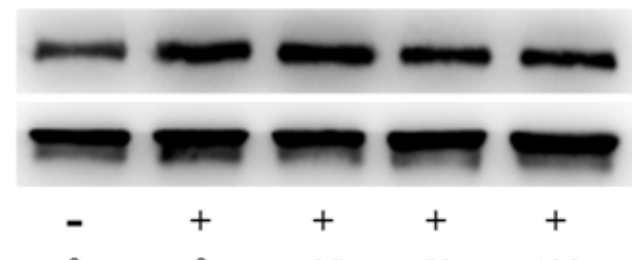

$25 \quad 50$

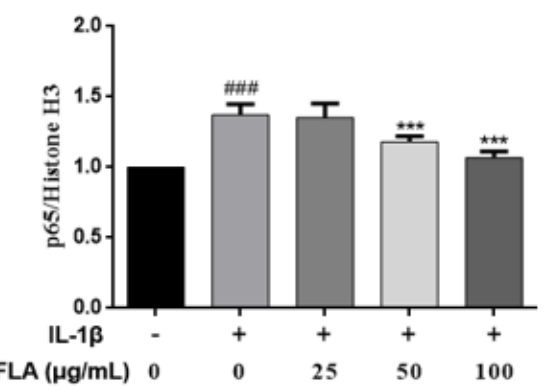

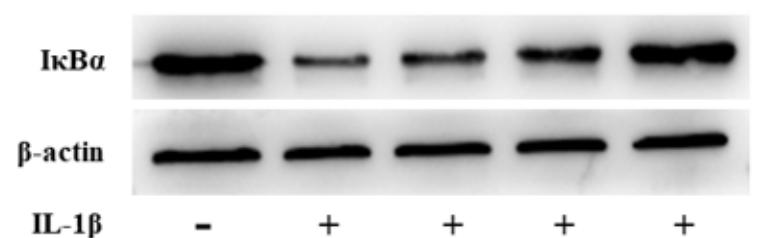

FLA $(\mu \mathrm{g} / \mathrm{mL})$

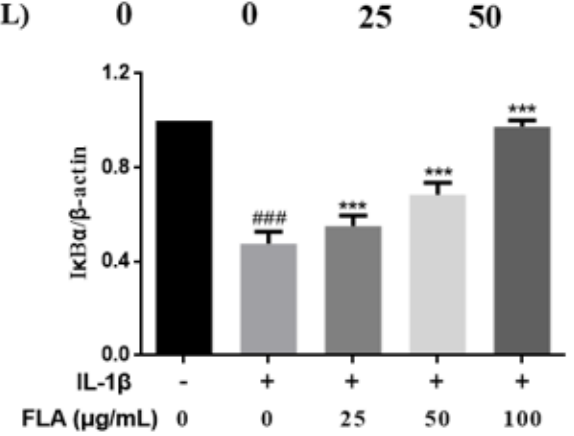

\section{Figure 6}

Effects of FLA on IL-1 $\beta$-induced phosphorylation of MAPKs and the activation of NF-KB in HFLS-RA. HFLS-RA were pretreated with various concentrations of FLA for $1 \mathrm{~h}$ and then exposed to IL-1 $\beta$ for another 24h. (A) The total and phosphorylated level of JNK, ERK1/2 and p38 were detected by Western blotting. (B) The nuclear protein and cytoplasmic protein were separated from the cells using Nuclear and Cytoplasmic Extraction Reagents. The translocation of NF-KB/p65 to the nucleus and the degradation of 
IKBa in the cytoplasm were measured by western blotting. Histone $\mathrm{H} 3$ and $\beta$-actin were used as loading controls for nuclear and cytoplasmic proteins, respectively. Values are expressed as means \pm SD of three independent experiments. \#\#\# $p<0.001 \mathrm{IL}-1 \beta$ vs control, $* * * p<0.001 \mathrm{IL}-1 \beta+\mathrm{FLA}$ vs IL-1 $\beta$ and * $p<0.05$ IL-1 $\beta$ + FLA vs IL-1 $\beta$.
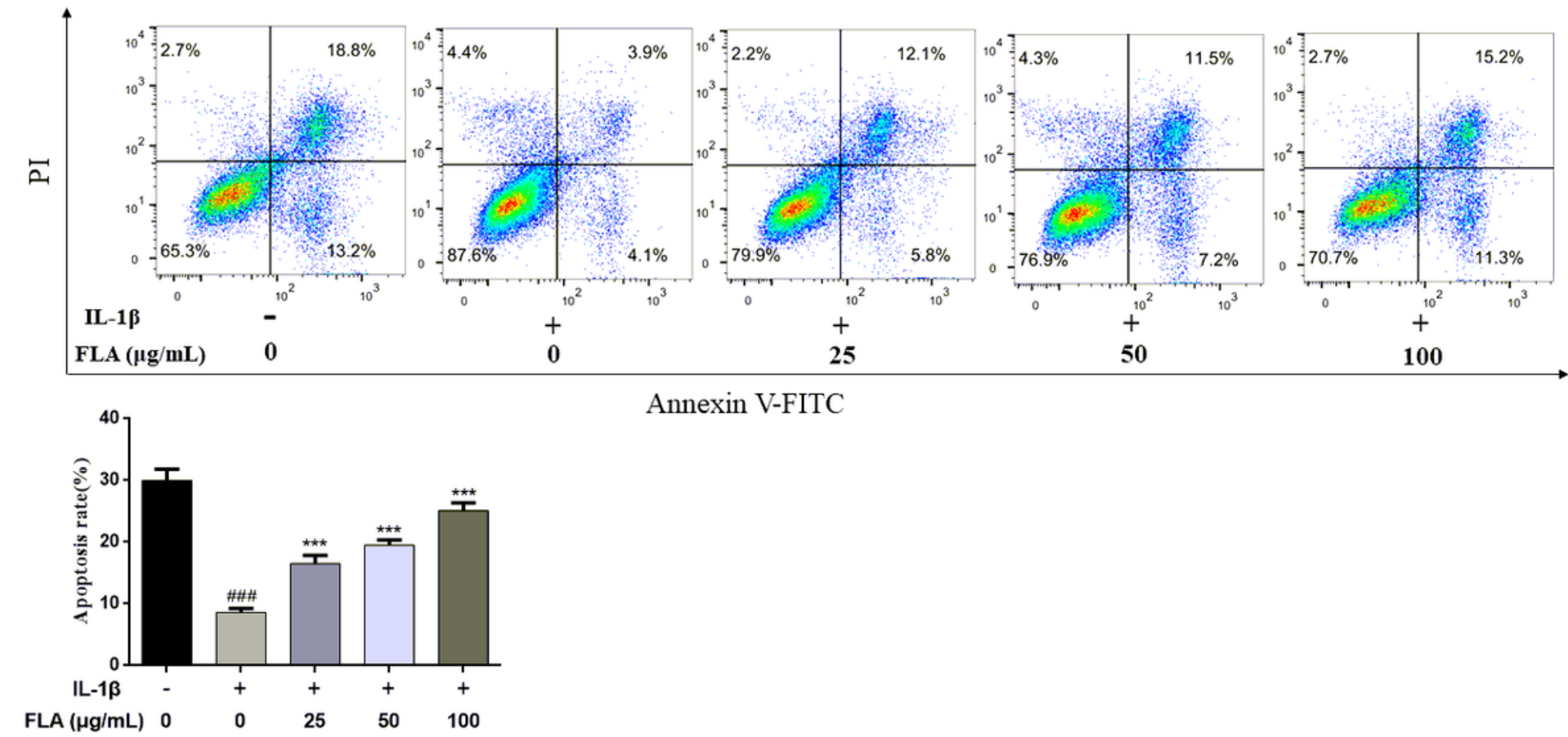

Figure 7

Effects of FLA on apoptosis of HFLS-RA. HFLS-RA were pretreated with various concentrations of FLA for $1 \mathrm{~h}$ and then exposed to IL-1 $\beta$ for another $24 \mathrm{~h}$. Apoptosis in HFLS-RA was detected by Annexin V-FITC/PI with flow cytometric analysis. Values are expressed as means $\pm S D$ of three independent experiments. $\# \# \# p<0.001 \mathrm{IL}-1 \beta$ vs control, $* \star \star p<0.001 \mathrm{IL}-1 \beta+$ FLA vs IL-1 $\beta$. 


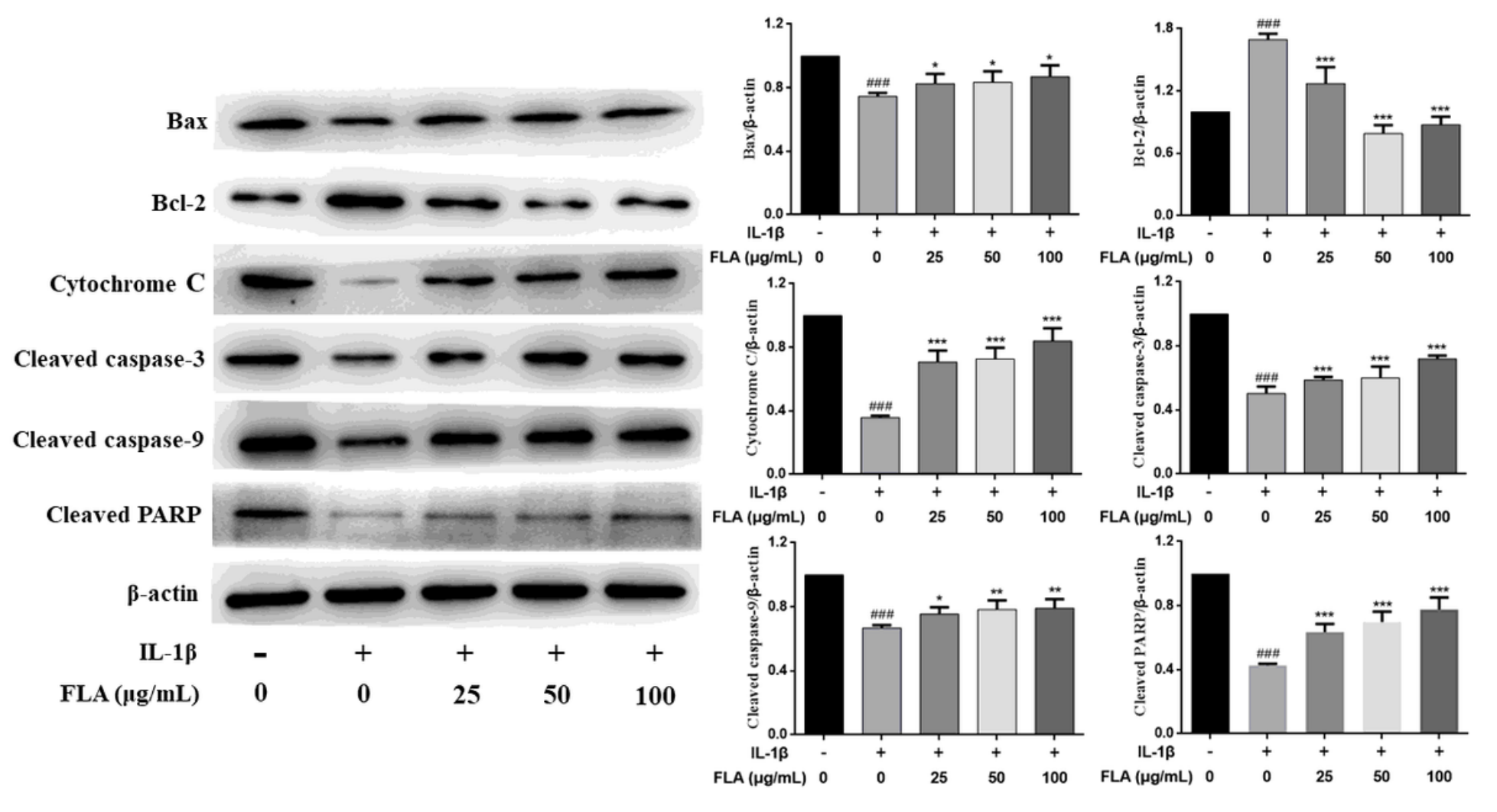

Figure 8

Effects of FLA on mitochondrial apoptotic pathway-related proteins in HFLS-RA. HFLS-RA were pretreated with various concentrations of FLA for $1 \mathrm{~h}$ and then exposed to IL-1 $\beta$ for another $24 \mathrm{~h}$. Western blotting was used to detect the expression of Bax, Bcl-2, Cytochrome C, Cleaved caspase 3, cleaved caspase 9 and Cleaved PARP in HFLS-RA. The Band intensities was normalized to $\beta$-actin. Values are expressed as means \pm SD of three independent experiments. \#\#\# $p<0.001 \mathrm{IL}-1 \beta$ vs control, $* \star \star ~ p<0.001 \mathrm{IL}-1 \beta+\mathrm{FLA}$ vs IL-1 $\beta$, ** $p<0.01 \mathrm{IL}-1 \beta+$ FLA vs IL-1 $\beta$ and * $p<0.05$ IL-1 $\beta+$ FLA vs IL-1 $\beta$. 